\title{
SOURCES OF ACOUSTIC SCATTERING IN NORMAL KIDNEYS
}

\author{
M.F. Insana*, T.J. Hall*, J.L. Fishback $\dagger$, G.G. Cox*, J.G. Wood $\neq$, L.Y. Cheung $\ddagger$, and S.J. Rosenthal*
}

Departments of * Radiology, †Pathology, and $\ddagger$ Surgery

Univ. of Kansas Medical Center, Kansas City, Kansas 66103

\begin{abstract}
Properties derived from acoustic backscatter measurements are examined to disclose sources of acoustic scattering in normal kidneys. We find that the backscatter intensity from kidney parenchyma may be modeled using a two-component Gaussian correlation function. These two components yield effective scatterer size estimates of $220 \pm 15 \mu \mathrm{m}$ at low frequencies and $55 \pm 10 \mu \mathrm{m}$ at high frequencies, which correspond to the average glomerular and renal tubule diameters, respectively. A separate study using test media verifies the ability of the method to distinguish between mixtures of two different scattering structures from backscatter measurements at different frequencies.
\end{abstract}

\section{INTRODUCTION}

A long-term goal of ultrasonic tissue characterization research is to identify the important structures in tissue that backscatter sound. Although backscatter measurements $[2,12]$ and acoustic microscopy data [6] have indicated that connective tissues, fat, and water are the elements of scattering sources in soft tissues, the complexity of tissue architectures has made it difficult to identify histological structures using acoustic backscatter.

In this paper, we combine acoustic and histologic measurements within the framework of single-scatter theory to identify the microscopic structures in kidney tissues that are most likely to scatter ultrasound. The experiments focus on explaining backscatter anisotropy, defined as the variation in acoustic backscatter that occurs when the angle of the incident sound field changes with respect to the orientation of aligned tissue structures. In the parenchyma of the kidney, nephrons and blood vessels provide this type of aligned structure.

\section{METHODS}

We adopt the empirical approach to the identification of scatterers, which involves comparing mathematical models for backscatter to measurements of backscatter. Derivations of the method, experimental designs, and verification using test media are described elsewhere $[4,5]$.

Models. Tissue is modeled as a medium containing randomly-positioned impedance fluctuation, i.e., scatterers. Structural properties of random media, such as the effective scatterer size, are often characterized by the correlation function for the medium. Using single-scatter theory, a linear relationship between the backscatter coefficient, $\sigma_{b}$, and the Fourier transform of the correlation function for tissue, which we call the intensity form factor $F$, may be expressed as

$$
\sigma_{b}(f)=C f^{4} D^{6} \bar{n} \gamma_{o}^{2} F_{\theta}(f, D),
$$

where $C$ is a constant, $f$ is frequency, $D$ is the effective scatterer size, and $\bar{n} \gamma_{o}^{2}$ is the effective scattering strength (i.e., product of the average number of scatterers per unit volume and the mean-square variation in acoustic impedance per scatterer). For situations where this simple scattering model is valid, the form factor for a given angle of incidence $\theta$ depends only on frequency and scatterer size. A Gaussian correlation model $[7,10,11,4]$ was selected to model renal tissues; therefore, $F_{\theta}(f, D)=\exp \left(-8.16 f^{2} D^{2}\right)$. For scatterers that are very small compared to the wavelength, $F$ is nearly constant and equal to one at all frequencies in the bandwidth. For larger scatterers, $F$ is a function the decreases with frequency and is equal to one at $0 \mathrm{MHz}$.

Measurements. Backscatter coefficients are measured for tissue samples in vitro using a broad-band substitution technique [5]. Time-gated rf echo signals are Fourier transformed to estimate a power spectral density. This result is divided by the power spectral density of a reference reflector and multiplied by factors describing attenuation in the tissue and the geometry of the experiment to determine $\sigma_{b}$. Form factors are estimated by dividing $\sigma_{b}$ by $f^{4}$ and scaling the result such that the measured form factor, extrapolated to zero frequency, is one.

Three Parameters. Three parameters are determined by comparing modeled $(F)$ and measured $(\hat{F})$ form factors. First, $F$ functions are computed for different scatterer sizes over a range of frequencies and 
stored in a look-up table. Second, each $F$ stored is numerically fit to $\hat{F}$ using least-squares analysis. Third, the model function that yields the smallest meansquare error when fit to $\hat{F}$ determines the effective scatterer size estimate $D$. Combining $D$ and Eq.(1), the scattering strength, $\bar{n} \gamma_{o}^{2}$, is estimated. Conceptually, $D$ is estimated from the frequency dependence of backscatter and $\vec{n} \gamma_{o}^{2}$ from the magnitude of backscatter. The method is most sensitive when the product of the wavenumber and the scatterer radius, $k a$, is between 0.5 and 1.2. Estimation procedures and limitations have been described $[4,5]$. A third parameter, the integrated backscatter coefficient $(I B C)$, is the frequency average of $\sigma_{b}$ over a specified bandwidth. This parameter has been applied extensively by Miller and colleagues [8] to study the microstructure of myocardium.

\section{MATERIALS}

Kidney Tissue. Excised dog kidneys obtained from unrelated experiments were scanned in saline at $19^{\circ} \mathrm{C}$. Dog kidneys are an excellent model for study because the acoustic properties and microscopic anatomy are very similar to the human kidney. Strips of the renal cortex were cut such that the nephron orientation was well defined. Samples were then cast in agar blocks to mark eight nephron orientations at $45^{\circ}$ increments. At $\theta=0^{\circ}$, the beam axis is parallel to the nephron orientation and incident upon the capsule side of the cortex. (The capsule was removed.) At $\theta=$ $180^{\circ}$, the beam axis was also parallel to the nephron, but incident upon the medulla side of the cortex. At $90^{\circ}$ and $270^{\circ}$, the beam axis was perpendicular to the nephrons, etc.

Test media. An agar sample containing a mixture of two different diameter distributions of glass microspheres was constructed. There were fewer of the large-diameter spheres (see right vertical scale in Fig. 1) than small (left vertical scale), and all the spheres were randomly positioned in the agar. The distribution of large spheres had a spike at $173 \mu \mathrm{m}$, where there were 0.099 spheres per $\mathrm{mm}^{3}$. The distribution of small spheres had a spike at $40 \mu \mathrm{m}$, where there were 18.7 spheres per $\mathrm{mm}^{3}$. Other sphere diameters were added to provide diversity in scatterer sizes to minimize sharp resonance peaks in backscatter observed with narrow-diameter distributions. Agar test samples of the two separate glass-sphere distibutions were also constructed. The speed of sound and attenuation in all three samples were approximately equal; the average values are $1534 \mathrm{~m} / \mathrm{s}$ and $0.031 f^{1.72} \mathrm{~dB} / \mathrm{cm}$, respectively. Measured backscatter coefficients are plotted for the three samples in figure 2 .

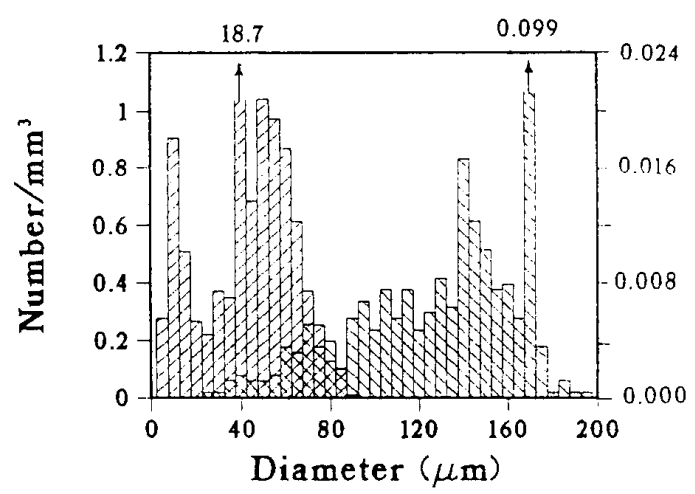

Fig. 1 Diameter distributions for the glass sphere-inagar test media are shown. The number per volume for the small-diameter spheres is indicated by the vertical axis on the left. The number per volume for the largediameter spheres is much smaller than the small spheres, as indicated by the vertical axis on the right. There is large spike in the small-scatterer distribution at $40 \mu \mathrm{m}$ and large-scatterer distribution at $173 \mu \mathrm{m}$.

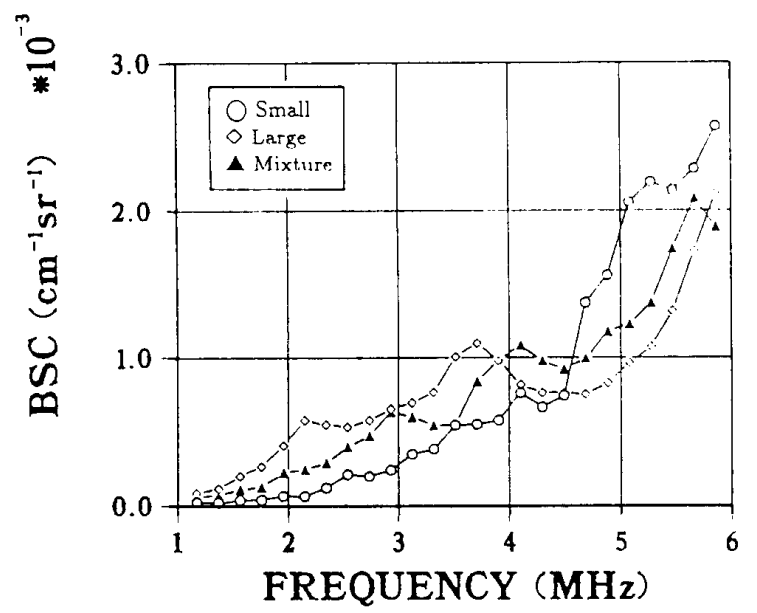

Fig. 2 Backscatter coefficients, $\sigma_{b}$, measured for the small and large diameter distributions of spheres (Fig.1) are plotted. Three sets of data are shown, two from samples of the individual distributions and one from the mixture. 


\section{RESULTS AND DISCUSSION}

Kidney Tissue. A form factor measured for one sample of renal cortex, at $0^{\circ}$ scanning angle (parallel incidence), is plotted in figure $3 \mathrm{a}$. Clearly, no single Gaussian model can adequately represent this data. However, the data is well represented by two Gaussian models if it is divided into two frequency ranges at $5 \mathrm{MHz}$. In this sample, the best-fit model corresponds to an effective scatterer size of $204 \mu \mathrm{m}$ below $5 \mathrm{MHz}$ and $71 \mu \mathrm{m}$ above $5 \mathrm{MHz}$. Averaging the results over samples from four kidneys yields an effective scatterer size for kidney cortex of $220 \pm 15 \mu \mathrm{m}$ at low frequencies and $55 \pm 10 \mu \mathrm{m}$ at high frequencies. $D$ estimates are approximately independent of scanning angle.

The form factor does vary systematically with scanning angle, particularly at high frequencies (Fig. 3b). The small-scatterer contribution (solid lines), relative to that for large scatterers (dashed line), oscillates sinusoidally with scanning angle, where it is maximum for perpendicular incidence and minimum for parallel incidence. Solid lines in figure $3 \mathrm{~b}$ represent the limits of the oscillatory behavior of the small-scatterer contribution. IBC values measured for high and low frequency bands also vary sinusoidally with angle. The ratios of maximum to minimum $\mathrm{IBC}$ are 2.4 at low frequency and 4.7 at high frequencies. Finally, patterns in the scattering strength versus scanning angle curve were seen to closely follow those for IBC.

The two backscatter components have their counterparts in histological observation. Using light microscopy, the cortical samples revealed an average glomerular diameter of $216 \pm 25 \mu \mathrm{m}$ and an average renal tubule and blood vessel diameter of $41 \pm 8 \mu \mathrm{m}$. The basement membranes in renal tubules, blood vessels, and glomeruli contain collagen, which provides an acoustic impedance fluctuation large enough to scatter sound. Therefore the data suggest that, in the renal cortex, glomeruli dominate the frequency dependence of backscatter at low frequencies, and renal tubules and blood vessels at high frequencies. The correlation between IBC and strength supports the theory that aligned segments of tubules and blood vessels are responsible for the anisotropy. Also, the convoluted tubules could provide enough nonaligned structure to produce angle independent $D$ estimates at high frequencies.

Nicholas [11] and Bamber [1] used multicomponent backscatter models to explained the frequency dependence of $\sigma_{b}$ for liver in terms of microscopic anatomy. In constrast to kidney, liver demonstrates isotropic acoustic properties $[3,9]$. Also several investigators suggest that an exponential correlation model is most appropriate for liver $[10,11]$.
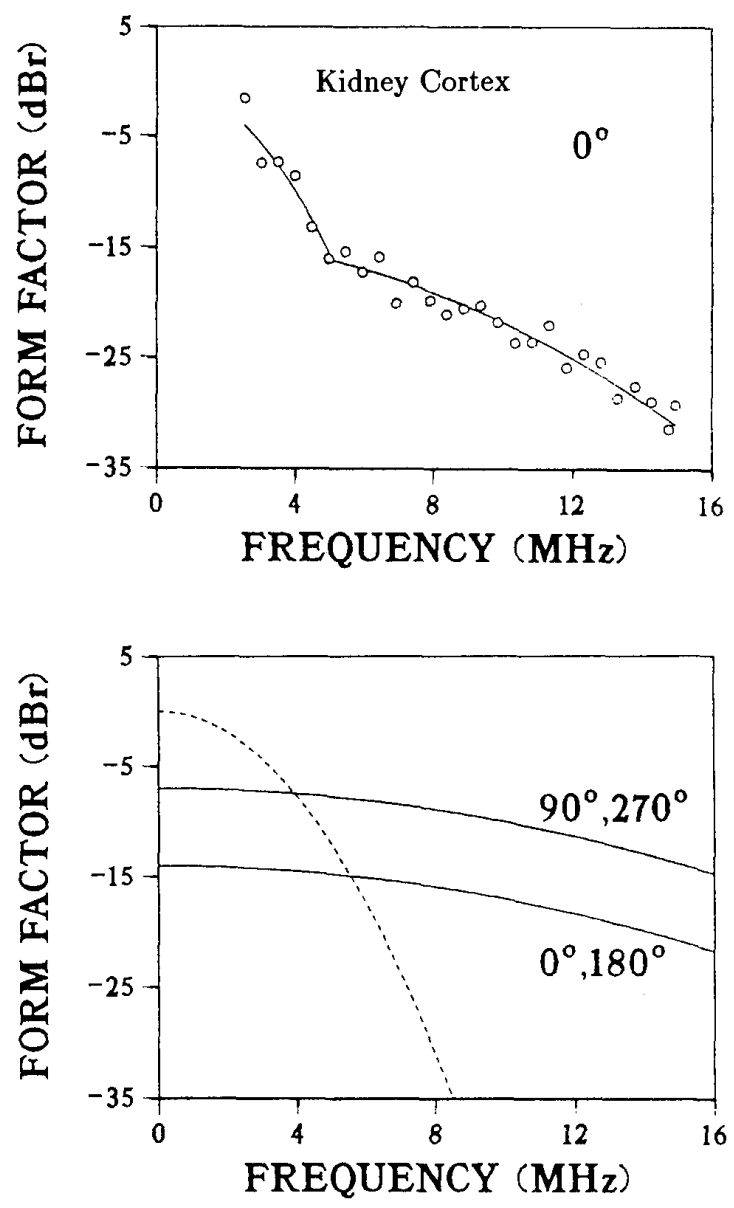

Fig. 3 (a) Form factors derived from $\sigma_{b}$ measurements for renal cortex at parallel incidence (points) and the corresponding "best-fit" Gaussian model functions (lines). Data indicate two components to backscatter, above and below $5 \mathrm{MHz}$. (b) Plot showing the decomposition of the form factor for kidney into large scatterers (dotted line) and small scatterers (solid lines), and how that varies with scanning angle. The magnitude of the small-scatterer contribution oscillates with scanning angle. It is largest for perpendicular incidence and smallest for parallel incidence. 
Test Media. A form factor measured for the twocomponent agar test sample is shown in figure 4 . The form factor may be divided into two components at $\sim 4 \mathrm{MHz}$, corresponding to the frequency where the $\sigma_{b}$ for the two components are approximately equal (See Fig. 2). Above $4 \mathrm{MHz}$ small spheres are the greatest contributor to backscatter, and below $4 \mathrm{MHz}$ large spheres are the greatest contributor. The effective scatterer size estimate is $201 \mu \mathrm{m}$ below $4 \mathrm{MHz}$ and $105 \mu \mathrm{m}$ above $4 \mathrm{MHz}$. Compared with the diameter distributions in figure 1 , these estimates are high for several reasons. As we showed previously, the Gaussian correlation model tends to overestimate glass sphere diameters, and the size estimate also depends on the width of the diameter distribution [5]. In addition, the large scatterers more clearly dominate scattering below $4 \mathrm{MHz}$ than the small scatterers do above $4 \mathrm{MHz}$. These details notwithstanding, the test-sample data serve to verify the ability of the method to distinguish between two scattering structures that are spatially mixed by an analysis of backscatter coefficients at different frequencies.

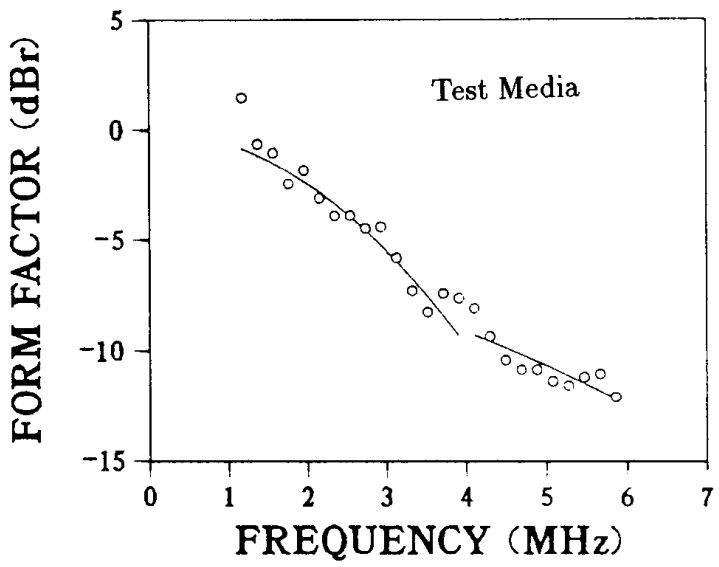

Fig. 4 Form factor measurements and "best-fit" Gaussian model functions for the mixture test medium described by figures 1 and 2 .

\section{CONCLUSIONS}

Acoustic backscatter from renal cortex is anisotropic. The anisotropy can be explained by describing backscatter using a two-component Gaussian correlation model. The two scattering components were found to correspond to structures in the microscopic anatomy. Data indicate that the principal scattering structures are the glomeruli below $\sim 5 \mathrm{MHz}$ and renal tubules and blood vessels above $\sim 5 \mathrm{MHz}$. The effective scatterer size indicates the average glomerular diameter independent of scanning angle in the lowfrequency range used for diagnostic imaging.

\section{References}

[1] Bamber J.C. (1979) Theoretical modelling of the acoustic scattering structure of human liver, Acoust. Lett. 3, 114-119.

[2] Bamber J.C., Hill C.R., and King J.A. (1981) Acoustic properties of normal and cancerous human liver - II. Dependence on tissue structure, Ultrasound in Med. E Biol. 7, 135-144.

[3] Campbell J.A. and Waag R.C. (1984) Measurements of calf liver ultrasonic differential and total scattering cross sections, J. Acoust. Soc. Am. 75 , 603-611.

[4] Insana M.F., Wagner, R.F., Brown D.G., and Hall T.J. (1990) Describing small-scale structure in random media using pulse-echo ultrasound, $J$. Acoust. Soc. Am. 87, 179-192.

[5] Insana M.F. and Hall T.J. (1990) Parametric ultrasound imaging from backscatter coefficient measurements: image formation and interpretation, Ultrasonic Imaging, 12, 245,267.

[6] Kessler L.W., Fields S.I. and Dunn F. (1974) Acoustic microscopy of mammalian kidney, $J$. Clin. Ultrasound 2, 317-320.

[7] Lizzi F.L., Ostromogilsky M., et al. (1987) Relationship of ultrasonic spectral parameters to features of tissue microstructure, IEEE Trans. Ultrason. Ferroelec. Freq. Contr. UFFC-34, 319329 .

[8] Miller J.G., Perez J.E., Mottley J.G., et al. (1983) Myocardial tissue characterization: an approach based on quantitative backscatter and attenuation, Proc. IEEE Ultrasonics Symposium Vol. 83CH1947-1, 782-793.

[9] Mottley J.G. and Miller J.G. (1990) Anisotropy of the ultrasonic attenuation in soft tissues: measurments in vitro, J. Acoust. Soc. Am. 88, 12031210.

[10] Nassiri D.K. and Hill C.R. (1986) The use of angular acoustic scattering measurements to estimate structural parameters of human and animal tissues, J. Acoust. Soc. Am 79, 2048-2054.

[11] Nicholas D (1982) Evaluation of backscattering coefficients for excised human tissues: results, interpretation, and associated measurements, Ultrasound Med. Biol. 8, 17-28.

[12] O'Donnell M., Mimbs J.W., and Miller J.G. (1981) Relationship between collagen and ultrasonic backscatter in myocardial tissue, $J$. Acoust. Soc. Am. 69, 580-588. 